\title{
INTERACTION OF A SHEAR WALL WITH THE SOIL FOR INCIDENT PLANE $S H$ WAVES: ELLIPTICAL RIGID FOUNDATION
}

\author{
By H. L. Wong and M. D. TRIfunaC
}

\begin{abstract}
The closed-form solution of the dynamic interaction of an elastic shear wall and the elastic homogeneous half-space, previously known only for the rigid foundation with circular cross section, has been generalized to apply for the foundation with elliptical cross section. It is shown that the interaction equation depends on the incidence angle of plane $S H$ waves and that this dependence gradually disappears as the elliptical cross section approaches the circular one. The effectiveness with which the rigid foundation can scatter the incident energy has been found to increase with the depth of the foundation.
\end{abstract}

\section{INTRODUCTION}

In spite of the fact that numerous structures have one or more stories below the ground level and that the foundation is in practically all cases well below the surface, only a few, simple soil-structure interaction cases having deep foundation have been reported so far in the literature (e.g., Luco, 1969; Novak, 1973; Tajimi, 1969; Trifunac, 1972; Thau, 1971). In most other studies of soil-structure interaction, the building foundation, often assumed to be a rigid plate, is bonded to the surface of the elastic half-space (e.g., Richart, et al., 1970; Oien, 1971; Luco and Westman, 1971). Although such geometry often significantly reduces the mathematical effort required for the formulation and solution of the problem, it may lead to unpredictable modifications of the final results, since the scattering and diffraction effected by the deep foundation are essentially eliminated by the very nature of the assumed model.

The purpose of this paper is, therefore. to study the effects that the depth of foundation may have on the overall soil-structure interaction problem and the extent to which these effects may depend on the direction of incident waves.

\section{THE MODEL}

The model studied here represents a logical extension of the model considered by Luco (1969) and Trifunac (1972). It consists of an infinitely long elastic shear wall of height $H$ and thickness $2 A$ or $2 b$, depending on whether we analyze the shallow (Figure la) or the deep (Figure 1b) foundation case, respectively. The rigidity and the velocity of shear waves in the isotropic and homogeneous wall are given by $\mu_{b}$ and $\beta_{b}$. This wall is erected on a rigid, infinitely long foundation beam, whose cross section corresponds to one half of an ellipse and whose major and minor axis dimensions are $A$ and $b$. The elastic and homogeneous half-space, which is assumed to be welded to the rigid foundation, is characterized by the rigidity $\mu$ and the velocity of shear waves $\beta$.

\section{InCident Ground Motion}

- We assume the excitation to consist of plane $S H$ waves with incident angle $\theta$, which is 
measured positive in the counterclockwise direction from the positive $x$ axis to the normal on the incident plane wave front (Figure 1a). Assuming the incident motion to be

$$
u_{z}^{i}=\exp \left[-i \omega\left(t-\frac{x}{c_{x}}-\frac{y}{c_{y}}\right)\right]
$$

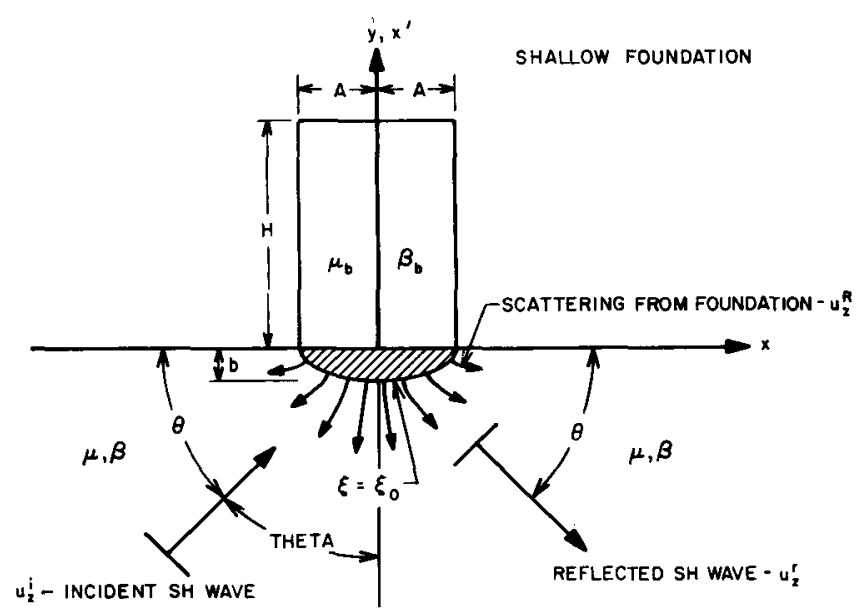

(a)

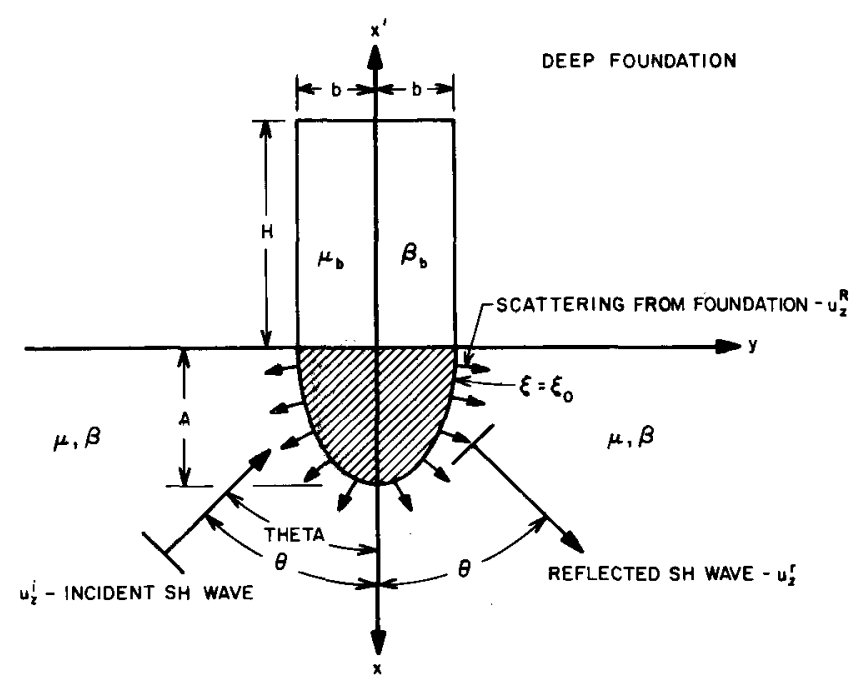

(b)

FIG. 1. Shear wall, foundation and soil.

where

$$
c_{x}=\beta / \cos \theta \quad \text { and } \quad c_{y}=\beta / \sin \theta,
$$

the resulting "free-field" motion, i.e. motion of the half-space in the absence of the structure and its foundation becomes 


$$
u_{z}{ }^{i}+u_{z}{ }^{r}=\exp \left[-i \omega\left(t-\frac{x}{c_{x}}\right)\right] 2 \cos \frac{\omega y}{c_{y}},
$$

where $u_{z}{ }^{r}$ is the wave reflected from the half-space boundary at $y=0$ (Figure 1a). For the rotated $x, y$ coordinate system in Figure $1 \mathrm{~b}$ the above results apply, provided $\pi-\theta$ is substituted for $\theta$ in (2).

\section{Wave Motion in the Half-Space}

The Helmholtz equation in cartesian coordinates for the $S H$ waves, $u_{z}$, in $y \leqq 0$ (Figure 1a) is

where

$$
\frac{\partial^{2} u_{z}}{\partial x^{2}}+\frac{\partial^{2} u_{z}}{\partial y^{2}}+k^{2} u_{z}=0
$$

$$
k=\omega / \beta \text {. }
$$

To transform this equation into the elliptic coordinates, we let

$$
\begin{array}{ll}
x=a \cosh \xi \cos \eta, & 0<\xi<\infty \\
y=a \sinh \xi \cos \eta, & 0<\eta<2 \pi
\end{array}
$$

where $a$ is the distance between the origin and the focus of an ellipse $\xi=\xi_{0}$. We then obtain

Letting

$$
\frac{\partial^{2} u_{z}}{\partial \xi^{2}}+\frac{\partial^{2} u_{z}}{\partial \eta^{2}}+a^{2} k^{2}\left[\cosh ^{2} \mu-\cos ^{2} \theta\right] u_{z}=0
$$

there follows

$$
u_{z}=H(\eta) Z(\xi)
$$

$$
\begin{aligned}
& \frac{d^{2} H}{d \eta^{2}}+(p-2 q \cos 2 \eta) H=0 \\
& \frac{d^{2} Z}{d \xi^{2}}-(p-2 q \cosh 2 \xi) Z=0
\end{aligned}
$$

where

$$
q=\frac{1}{4} a^{2} k^{2}
$$

Equation (9) is called the Mathieu's equation, while (10) is called the modified Mathieu's equation, since for $\xi=-i \eta$ it reduces to (9). $p$ is the characteristic value.

The periodic solutions of (9) are

and

$$
c e_{2 m}(\eta, q), s e_{2 m+2}(\eta, q) \quad(\text { periodic with period } \pi \text { ) }
$$

$$
\left.c e_{2 m+1}(\eta, q), s e_{2 m+1}(\eta, q) \quad \text { (periodic with period } 2 \pi\right) .
$$

Solutions of the modified equation (10) are: $M c_{2 m}^{(1)}, M s_{2 m+2}^{(1)}, M c_{2 m+1}^{(1)}$ and $M s_{2 m+1}^{(1)}$. These become Bessel functions when $q \rightarrow 0$. Also, we have $M c_{2 m}^{(2)}, M s_{2 m+2}^{(2)}, M c_{2 m+1}^{(2)}$, $M s_{2 m+1}^{(2)}$ which become Neumann functions $Y_{2 m}$ as $q \rightarrow 0$. A linear combination of the above functions is also a solution

$$
\begin{aligned}
& M c_{2 m}^{(3)}=M c_{2 m}^{(1)}+i M c_{2 m}^{(2)} \\
& M c_{2 m}^{(4)}=M c_{2 m}^{(1)}-i M c_{2 m}^{(2)} .
\end{aligned}
$$

As $q \rightarrow 0, M c_{2 m}^{(3)} \rightarrow$ Hankel function of the first kind and $M c_{2 m}^{(4)} \rightarrow$ Hankel function of the second kind (Abramovitz and Stegun, 1970; Meixner, 1954). 
Consider now the case of a shallow foundation, the solution of the half-space problem excited by the plane $S H$-waves $u_{z}{ }^{i}+u_{z}{ }^{r}$ [given by (3)] can then be expressed in an infinite series of Mathieu functions as follows

$$
\begin{aligned}
u_{z}^{i}+u_{z}^{r}= & 4 \sum_{m=0}^{\infty}(-1)^{m} c e_{2 m}(\eta, q) M c_{2 m}^{(1)}(\xi, q) c e_{2 m}(\theta, q) \\
& +4 i \sum_{m=0}^{\infty}(-1)^{m} c e_{2 m+1}(\eta, q) M c_{2 m+1}^{(1)}(\xi, q) c e_{2 m+1}(\theta, q) .
\end{aligned}
$$

The wave scattered from and diffracted around the foundation, $u_{z}{ }^{R}$, can be written in the form

$$
u_{z}^{R}=\sum_{m=0}^{\infty}\left\{a_{2 m} M c_{2 m}^{(3)}(\xi, q) c e_{2 m}(\eta, q)+b_{2 m+1} M c_{2 m+1}^{(3)}(\xi, q) c e_{2 m+1}(\eta, q)\right\}
$$

where $a_{2 m}$ and $b_{2 m+1}$ are constants and it satisfies the boundary conditions

$$
\frac{\partial u_{z}{ }^{R}}{\partial \eta}=0 \quad \text { at } \quad \eta=0,-\pi
$$

and

$$
u_{z}{ }^{R} \rightarrow 0 \quad \text { as } \quad \xi \rightarrow \infty .
$$

The boundary condition at the foundation-soil interface is

$$
u_{z}=\Delta e^{-i \omega t} \quad \text { at } \quad \xi=\xi_{0},
$$

where $\Delta$ is the amplitude of motion of the rigid foundation. Using (12) and (13) to express $u_{z}=u_{z}{ }^{i}+u_{z}{ }^{r}+u_{z}{ }^{R}$ and matching it with (15) at $\xi=\xi_{0}$ gives

$$
\begin{aligned}
\Delta= & \sum_{m=0}^{\infty}\left[4(-1)^{m} c e_{2 m}(\eta, q) M c_{2 m}^{(1)}\left(\xi_{0}, q\right) c e_{2 m}(\theta, q)+\right. \\
& \left.+4(-1)^{m} i c e_{2 m+1}(\eta, q) M c_{2 m+1}^{(1)}\left(\xi_{0}, q\right) c e_{2 m+1}(\theta, q)\right]+ \\
& +\sum_{m=0}^{\infty}\left[a_{2 m} M c_{2 m}^{(3)}\left(\xi_{0}, q\right) c e_{2 m}(\eta, q)\right. \\
& \left.+b_{2 m+1} M c_{2 m+1}^{(3)}\left(\xi_{0}, q\right) c e_{2 m+1}(\eta, q)\right] .
\end{aligned}
$$

To use the orthogonality properties of $c e_{m}(\eta, q)$ we express $\Delta$ as $\Delta \cdot 1$ in a series of Mathieu functions. Letting

$$
1=\sum_{m=0}^{\infty}\left(\alpha_{2 m} c e_{2 m}+\beta_{2 m+1} c e_{2 m+1}+f_{2 m} s e_{2 m}+g_{2 m+1} s e_{2 m+1}\right)
$$

where

$$
\begin{gathered}
\alpha_{2 m}=\frac{1}{\pi} \int_{0}^{2 \pi} c e_{2 m} d \eta=2 \frac{1}{2 \pi} \int_{0}^{2 \pi} c e_{2 m} d \eta \equiv 2 A_{0}^{2 m} \\
\beta_{2 m+1}=\frac{1}{\pi} \int_{0}^{2 \pi} c e_{2 m+1} d \eta=0 \\
f_{2 m}=\frac{1}{\pi} \int_{0}^{2 \pi} s e_{2 m} d \eta=0 \\
g_{2 m+1}=\frac{1}{\pi} \int_{0}^{2 \pi} s e_{2 m+1} d \eta=0
\end{gathered}
$$


there follows

$$
\Delta=\Delta 2 \sum_{m=0}^{\infty} A_{0}^{2 m} c e_{2 m}(\eta, q) .
$$

As $q \rightarrow 0$, i.e. as the ellipse approaches a circle

and

$$
\begin{aligned}
A_{0}{ }^{0} \rightarrow \frac{\sqrt{ } 2}{2} ; c e_{0} \rightarrow \frac{\sqrt{ } 2}{2} ; A_{0}^{2 m} & \rightarrow 0 \\
m & \neq 0
\end{aligned}
$$

$$
\Delta=\Delta
$$

Thus, all higher harmonics are lost when $q \rightarrow 0$.

By the orthogonality of Mathieu functions, equation (16) may be written as

$$
\begin{gathered}
2 \Delta A_{0}^{2 m}=4(-1)^{m} M c_{2 m}^{(1)}\left(\xi_{0}, q\right) c e_{2 m}(\theta, q)+a_{2 m} M c_{2 m}^{(3)}\left(\xi_{0}, q\right) \\
0=4 i(-1)^{m} c e_{2 m+1}(\theta, q) M c_{2 m+1}^{(1)}\left(\xi_{0}, q\right)+b_{2 m+1} M c_{2 m+1}^{(3)}\left(\xi_{0}, q\right) .
\end{gathered}
$$

Equation (18) then gives

$$
\begin{aligned}
& a_{2 m}=\frac{2 A_{0}{ }^{2 m} \Delta-4(-1)^{m} M c_{2 m}^{(1)}\left(\xi_{0}, q\right) c e_{2 m}(\theta, q)}{M c_{2 m}^{(3)}\left(\xi_{0}, q\right)} \\
& b_{2 m}=-\frac{4 i(-1)^{m} M c_{2 m+1}^{(1)}\left(\xi_{0}, q\right) c e_{2 m+1}(\theta, q)}{M c_{2 m+1}^{(3)}\left(\xi_{0}, q\right)} .
\end{aligned}
$$

We note that all $a_{2 m}$ are dependent on $\Delta$ and $A_{0}{ }^{2 m}$, in contrast to the case for the foundation with circular cross section, where only the corresponding $a_{0}$ depends on $\Delta$ (Trifunac, 1972).

\section{Motion of The Shear Wall}

Although this part of the present analysis is identical to the corresponding portions of the previous papers by Luco (1969) and Trifunac (1972), we outline the principal steps of the derivation for completeness in this presentation.

With the differential equation of motion of a one-dimensional shear wall

$$
\frac{\partial^{2} u_{z}}{\partial x^{\prime 2}}=\frac{1}{\beta_{b}{ }^{2}} \frac{\partial^{2} u_{z}}{\partial t^{2}} ; \quad 0 \leqq x^{\prime} \leqq H
$$

and the boundary conditions

$$
\begin{array}{ccc}
\sigma_{x z}=\mu_{b} \frac{\partial u_{z}}{\partial x^{\prime}}=0 & \text { at } & x^{\prime}=H \\
u_{z}=\Delta e^{-i \omega t} & \text { at } & x^{\prime}=0
\end{array}
$$

the displacement $u_{z}$ of the shear wall is given by

where

$$
u_{z}=\Delta e^{-i \omega t}\left[\cos k_{b} x^{\prime}+\tan k_{b} H \sin k_{b} x^{\prime}\right]
$$

$$
k_{b}=\omega / \beta_{b} \text {. }
$$

The base shear force per unit length of the wall $f_{z}{ }^{b}$ is (Luco, 1969)

$$
f_{z}^{b}=-\omega^{2} M_{b}\left(\frac{\tan k_{b} H}{k_{b} H}\right) \Delta e^{-i \omega t}
$$


where

$$
M_{b}=\rho_{b} 2 a H .
$$

The natural frequencies of a shear wall on the rigid foundation are given by

$$
k_{b} H=(2 n+1) \frac{\pi}{2} ; \quad n=1,2,3, \ldots
$$

\section{INTERACTION}

The movement $\Delta e^{-i \omega t}$ of the foundation may be determined by writing the equation of motion for a rigid foundation

$$
-\omega^{2} M_{0} \Delta e^{-i \omega t}=-\left(f_{z}^{s}+f_{z}^{b}\right)
$$

where $M_{0}$ is the mass per unit length of the foundation. The $f_{z}^{s}$ is given by

where

$$
f_{z}^{s}=-\left.\int_{-\pi}^{0} \sigma_{\xi z}\right|_{\xi=\xi_{0}} d s
$$

$$
d s=\frac{A}{\cosh \xi_{0}}\left(\cosh ^{2} \xi_{0}-\cos ^{2} \eta\right)^{1 / 2} d \eta
$$

and

so that

$$
\left.\sigma_{\xi z}\right|_{\xi=\xi_{0}}=\left.\frac{\mu}{\frac{A}{\cosh \xi_{0}}\left(\cosh ^{2} \xi_{0}-\cos ^{2} \eta\right)^{1 / 2}} \cdot \frac{\partial u_{z}}{\partial \xi}\right|_{\xi=\xi_{0}}
$$

Now

$$
f_{z}^{s}=-\left.\int_{-\pi}^{0} \mu \frac{\partial u_{z}}{\partial \xi}\right|_{\xi=\xi_{0}} d \eta
$$

$$
\begin{aligned}
\left.\mu \frac{\partial u_{z}}{\partial \xi}\right|_{\xi=\xi_{0}}= & \mu\left\{\sum_{m=0}^{\infty}\left[a_{2 m} M c_{2 m}^{(3)}\left(\xi_{0}, q\right)+4(-1)^{m} c e_{2 m}(\theta, q) M c_{2 m}^{(1)}\left(\xi_{0}, q\right)\right] c e_{2 m}(\eta, q)\right. \\
& +\sum_{m=0}^{\infty}\left[b_{2 m+1} M c_{2 m+1}^{(3)}\left(\xi_{0}, q\right)\right. \\
& \left.\left.+4(-1)^{m} i c e_{2 m+1}(\theta, q) M c_{2 m+1}^{(1)}\left(\xi_{0}, q\right)\right] c e_{2 m+1}(\eta, q)\right\}
\end{aligned}
$$

with "," indicating the derivative with respect to $\xi$. Using the integrals

(32) becomes

$$
\begin{aligned}
& \int_{-\pi}^{0} c e_{2 m}(\eta, q) d \eta=\pi A_{0}^{2 m} \\
& \int_{-\pi}^{0} c e_{2 m+1}(\eta, q) d \eta=0
\end{aligned}
$$

$$
f_{z}^{s}=-\mu \pi\left\{\sum_{m=0}^{\infty}\left[a_{2 m} M c_{2 m}^{(3)}\left(\xi_{0}, q\right)+4(-1)^{m} c e_{2 m}(\theta, q) M c_{2 m}^{(1),}\left(\xi_{0}, q\right)\right] A_{0}{ }^{2 m}\right\} .
$$

The substitution of (25) and (36) into (28) yields

$$
\begin{aligned}
\mu \pi \sum_{m=0}^{\infty}\left\{a_{2 m} M c_{2 m}^{(3)}\left(\xi_{0}, q\right)+4(-1)^{m} c e_{2 m}(\theta, q) M c_{2 m}^{(1)}\left(\xi_{0}, q\right)\right\} A_{0}{ }^{2 m} \\
=-\omega^{2}\left[M_{0}+M_{b}\left(\frac{\tan k_{b} H}{k_{b} H}\right)\right] \Delta .
\end{aligned}
$$


Rearranging the parameters so that

where

$$
\frac{\mu \pi}{\omega^{2}} \frac{1}{\left[M_{0}+M_{b}\left(\frac{\tan k_{b} H}{k_{b} H}\right)\right]} \equiv \frac{1}{\frac{k^{2} b A}{2}\left[\frac{M_{0}}{M_{s}}+\frac{M_{b}}{M_{s}}\left(\frac{\tan k_{b} H}{k_{b} H}\right)\right]},
$$

$$
M_{s} \equiv \frac{\pi A b}{\rho 2}
$$

is the weight of soil replaced by the foundation and $A$ and $b$ are the major and minor axes of an ellipse, and by using (19), we get the final expression for $\Delta$

$$
\Delta=\frac{\sum_{m=0}^{\infty} 4(-1)^{m} A_{0}{ }^{2 m} c e_{2 m}(\theta, q)\left[\frac{M c_{2 m}^{(3)}\left(\xi_{0}, q\right)}{M c_{2 m}^{(3)}\left(\xi_{0}, q\right)} M c_{2 m}^{(1)}\left(\xi_{0}, q\right)-M c_{2 m}^{(1)},\left(\xi_{0}, q\right)\right]}{\frac{k^{2} b A}{2}\left[\frac{M_{0}}{M_{s}}+\frac{M_{b}}{M_{s}}\left(\frac{\tan k_{b} H}{k_{b} H}\right)\right]+\sum_{m=0}^{\infty} 2\left(A_{0}{ }^{2 m}\right)^{2}\left[\frac{M c_{2 m}^{(3) \prime}\left(\xi_{0}, q\right)}{M c_{2 m}^{(3)}\left(\xi_{0}, q\right)}\right]} .
$$

In the limit when $q \rightarrow 0$, i.e., when the ellipse approaches the circle, we have

$$
\begin{aligned}
& M c_{2 m}^{(1)}\left(\xi_{0}, q\right) \rightarrow J_{2 m}(k A) \\
& M c_{2 m}^{(3)}\left(\xi_{0}, q\right) \rightarrow H_{2 m}^{(1)}(k A) .
\end{aligned}
$$

Also for

and

$$
\begin{aligned}
m \neq 0 \quad c e_{2 m}(\theta, q) & \rightarrow \cos 2 m \theta \\
q & \rightarrow 0 \\
m=0 \quad c e_{0}(\theta, q) & \rightarrow \frac{1}{\sqrt{ } 2} \rightarrow A_{0}{ }^{\circ} \\
q & \rightarrow 0
\end{aligned}
$$

$$
A_{0}{ }^{2 m} \rightarrow \begin{cases}A_{0}{ }^{0}=\frac{1}{\sqrt{2}}, & m=0 \\ 0 & m \neq 0 .\end{cases}
$$

Thus, when $q \rightarrow 0, \Delta$ tends io

$$
\Delta_{q \rightarrow 0} \rightarrow \frac{2\left[J_{1}(k A)-\frac{J_{0}(k A) H_{1}{ }^{(1)}(k A)}{H_{0}{ }^{(1)}(k A)}\right.}{\frac{k A}{2}\left(\frac{M_{0}}{M_{S}}+\frac{M_{b}}{M_{S}} \frac{\tan k_{b} H}{k_{b} H}\right)-\frac{H_{1}{ }^{(1)}(k A)}{H_{2}^{(1)}(k A)}}
$$

and the $\theta$ dependence of $\Delta$ is lost (Trifunac, 1972). For large $q$ the $\theta$ dependence of $\Delta$ becomes important, especially when $b / A$ is small.

To characterize the problem in terms of dimensionless parameters we define

$$
\varepsilon \equiv \frac{k_{b} H}{k A}=\frac{\beta H}{\beta_{b} A} \text {. }
$$

It is seen that the flexible, slender and/or tall shear beams will be described by large values of $\varepsilon$.

In the above analysis, the coordinate system and the shallow foundation case shown in Figure 1(a) have been selected as an example. However, Mutatis mutandis it is readily seen that the same results hold for the deep foundation case, shown in Figure 1(b), provided 
$\pi-\theta$ is substituted for $\theta$ in (2) and in the subsequent equations. Thus, with the angles of incident waves as defined in Figures 1(a) and 1(b), the same solution can be used to study the corresponding vibrations of the shallow and deep foundations that have the same $b / A$ ratios. The same parameter $\varepsilon$ applies to both shallow and deep foundations.

Figures 2 through 6 show the amplitude of foundation motion $|\Delta|$ plotted versus $\omega A / \beta$, and for the angle of incident $S H$-waves "THETA" equal to $\pi-\theta$ for a shallow foundation (Figure 1a) and corresponding to $\theta$ for the deep foundation (Figure 1b). It is seen that for $\omega A \mid \beta=0$, all curves tend to 2 which is the amplification at the half-space boundary. Each figure presents $|\Delta|$ for the four axis ratios $b / A$ equal to $0.05,0.30,0.70$, and 0.99 . The small axis ratio $b / A$ represents either a shallow foundation (Figure 1a) or very deep foundation (Figure $1 b$ ), while the ratio $b / A=1$ corresponds to the semicircular cross section. Since the results for THETA $=0$ in the case of the shallow foundation (Figure 1a) are the same as those for THETA $=90^{\circ}$ for the deep foundation (Figure 1b), all the curves in Figures 2 through 6 are labeled first for shallow and then for the deep (in the brackets) foundation case. In each figure the data are plotted for the same $\varepsilon$ value ranging from 0 (Figure 2) to 8 (Figure 6) and for three values of $M_{b} / M_{S}=0.5,2.0$, and 8.0. For all calculations in this paper we have assumed that $M_{0} / M_{S}=1$.

Figure 2 presents the $\varepsilon=0$ case. From (41) it is seen that this value of $\varepsilon$ may be realized for a rigid wall $\left(\beta_{b}=\infty\right)$ or for a building mass concentrated at $x^{\prime}=0$ so that $H=0$. In either case, there is no motion of the building mass relative to the foundation, and consequently no building resonances enter into the problem. As a result, Figure 2 has no zeroes in the $|\Delta|$ diagrams, while all the other figures for $\varepsilon \neq 0$ have one or more equally spaced zero $\Delta \mid$ amplitudes. The zeroes in $|\Delta|$ versus $\omega A \mid \beta$ diagrams correspond to the natural frequencies of the shear wall and occur at $k_{b} H=\left(n+\frac{1}{2}\right) \pi$. This, of course, corresponds to $\omega A / \beta=\left(n+\frac{1}{2}\right) \pi / \varepsilon$.

The $\varepsilon=0$ case in Figure 2 describes the case of a rigid mass $M=M_{0}+M_{b}$ forced to vibrate by the incident $S H$ waves. It is seen from Figure 2 that for the small axis ratio, small $M_{b} / M_{S}$ ratio of 0.5 , the dimensionless frequency band for $\omega A / \beta$ between 0 and 3, very shallow foundation and vertical-wave incidence, or for very deep foundation and horizontal incidence, the foundation moves essentially like the half-space would move in the absence of any foundation. In these two cases the "projection" of that part of the foundation mass which is in contact with the half-space onto the normal of the planewave front is "small" relative to the cases of THETA $=90^{\circ}$ incidence for shallow foundation and THETA $=0^{\circ}$ incidence for deep foundations, so that the incident waves do not "see" the foundation very well. Consequently, the scattering and diffraction effects and interaction are therefore reduced. As the angle THETA increases toward $90^{\circ}$ for the shallow foundation, or decreases toward $0^{\circ}$ for the deep foundation, the size of the projection of the foundation onto the normal of the plane-wave front increases, the scattering and diffraction become more prominent, and $|\Delta|$ becomes more sensitive to changes of $\omega A / \beta$. This is best displayed in Figure 2 for $M_{b} / M_{S}=0.5$ and axis ratio equal to 0.05 . As the axis ratio increases toward 1, i.e., the elliptical cross section of the foundation tends toward a circular cross section, the size of the "projection" of the foundation onto the plane-wave front and the amplitude $|\Delta|$ become independent of the incidence angle THETA. For the axis ratio of 0.99 this dependence is already lost.

When $M_{b} / M_{S}$ is small or zero, and since we take $M_{0} / M_{S}=1$, the Figure 2 reflects the consequences of assuming that the foundation medium is rigid. As $M_{b} / M_{S}$ increases, the effective density of the foundation block increases relative to the density of the surrounding medium and the contribution of inertial forces becomes more prominent. The result of this is that the characteristics of a single-degree-of-freedom system represented by a spring, mass, and a dash-pot emerge as representative of the $|\Delta|$ curves in Figure 2. Keeping the foundation shape constant in effect means that one keeps the equivalent 

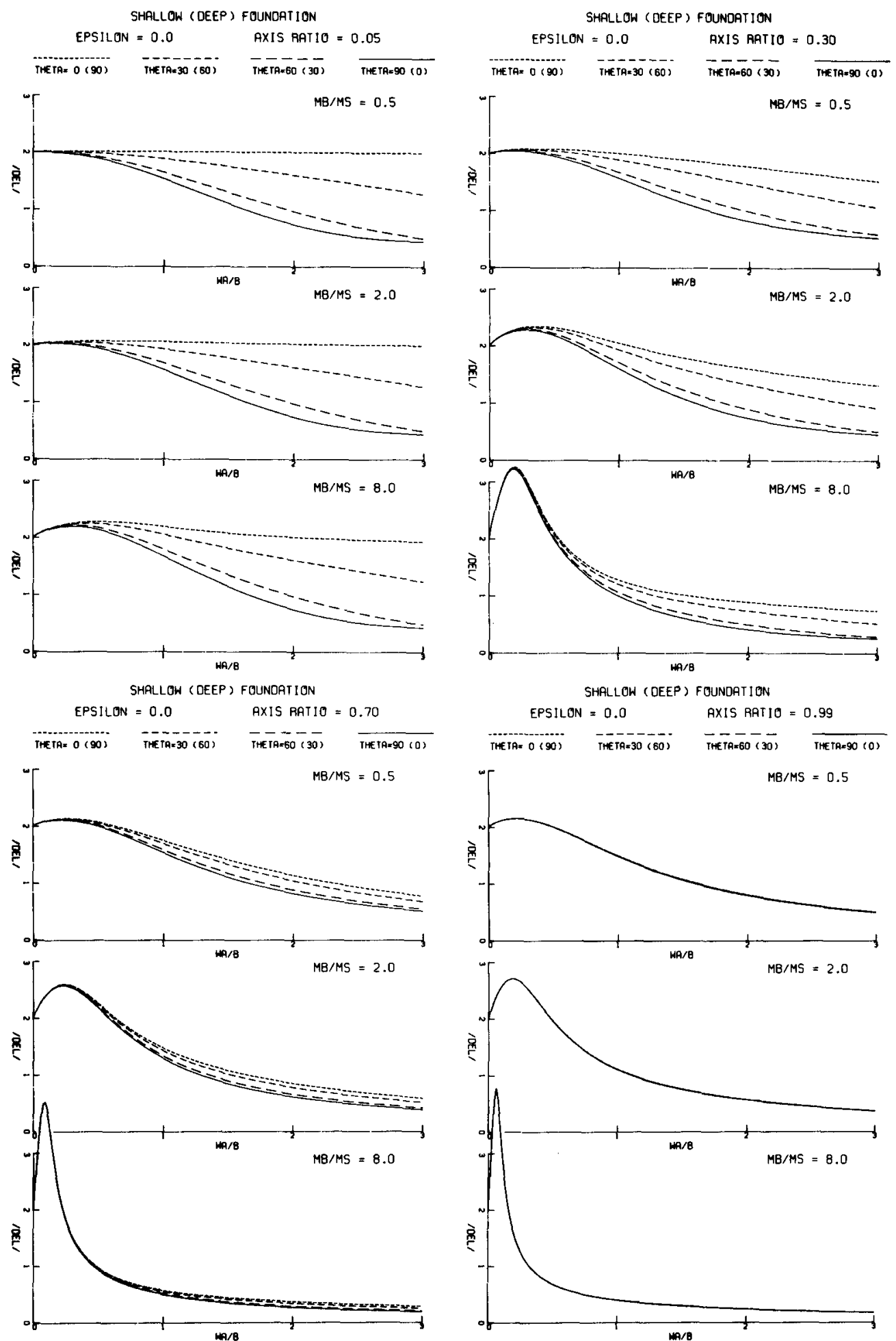

FIG. 2. Effect of interaction on the motion $|\Delta|$ of the rigid foundation. 

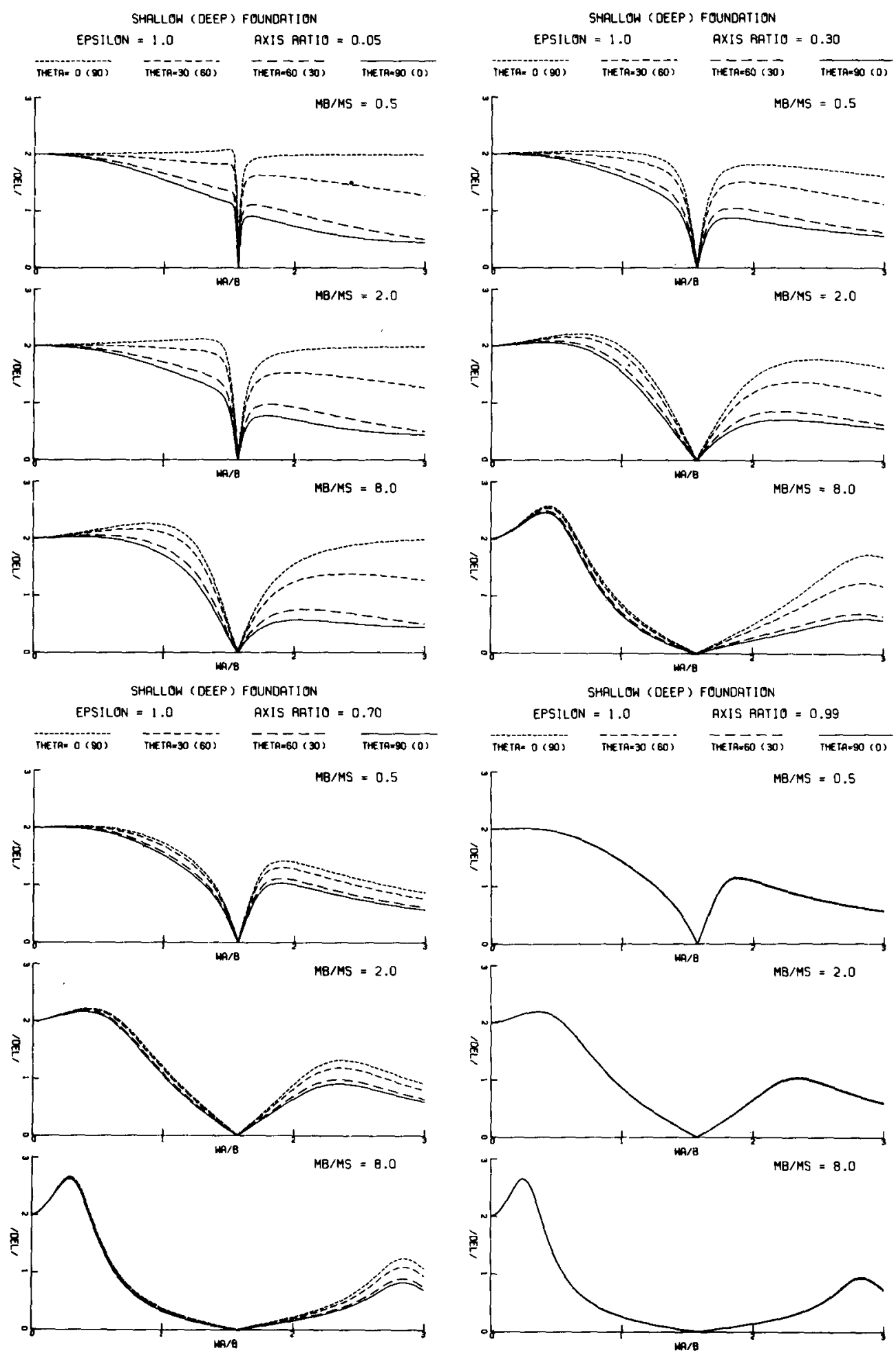

FIG. 3. Effect of interaction on the motion $|\Delta|$ of the rigid foundation. 

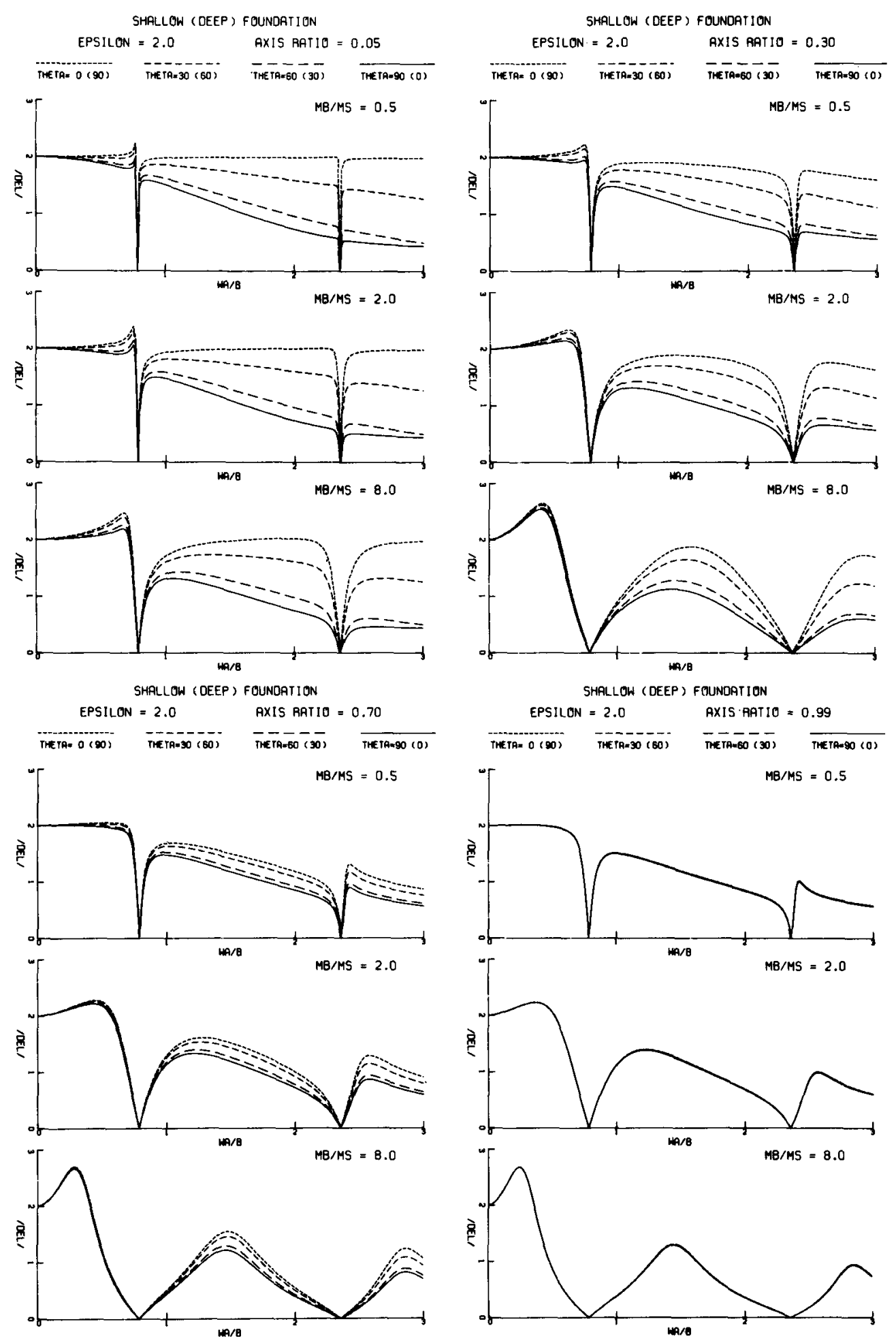

Fig. 4. Effect of interaction on the motion $|\Delta|$ of the rigid foundation. 

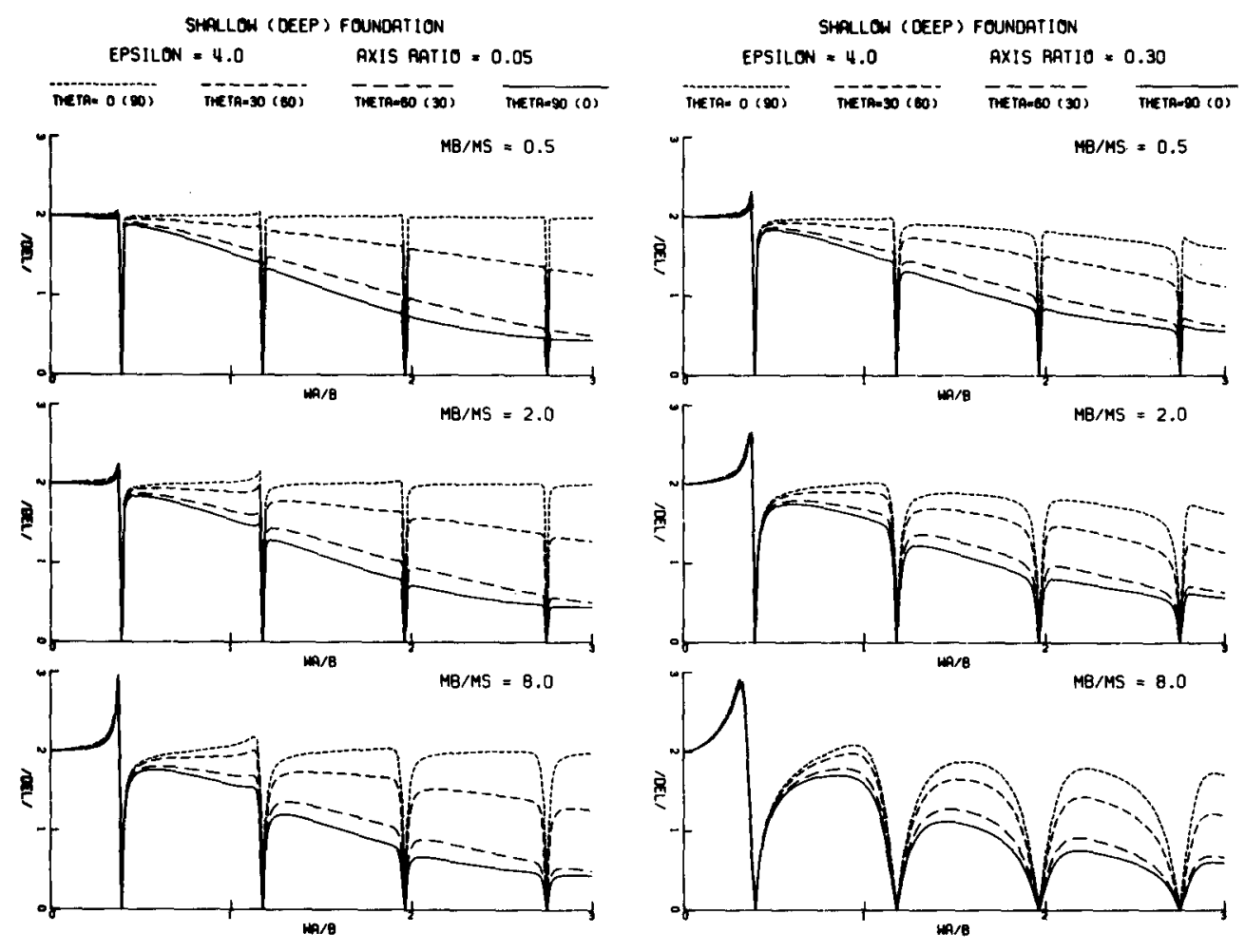

SHALLOW (DEEP) FOUNDATION

SHALLOW (DEEP) FOLNDATION

EPSILON $=4.0 \quad$ AXIS RATIO $=0.70$
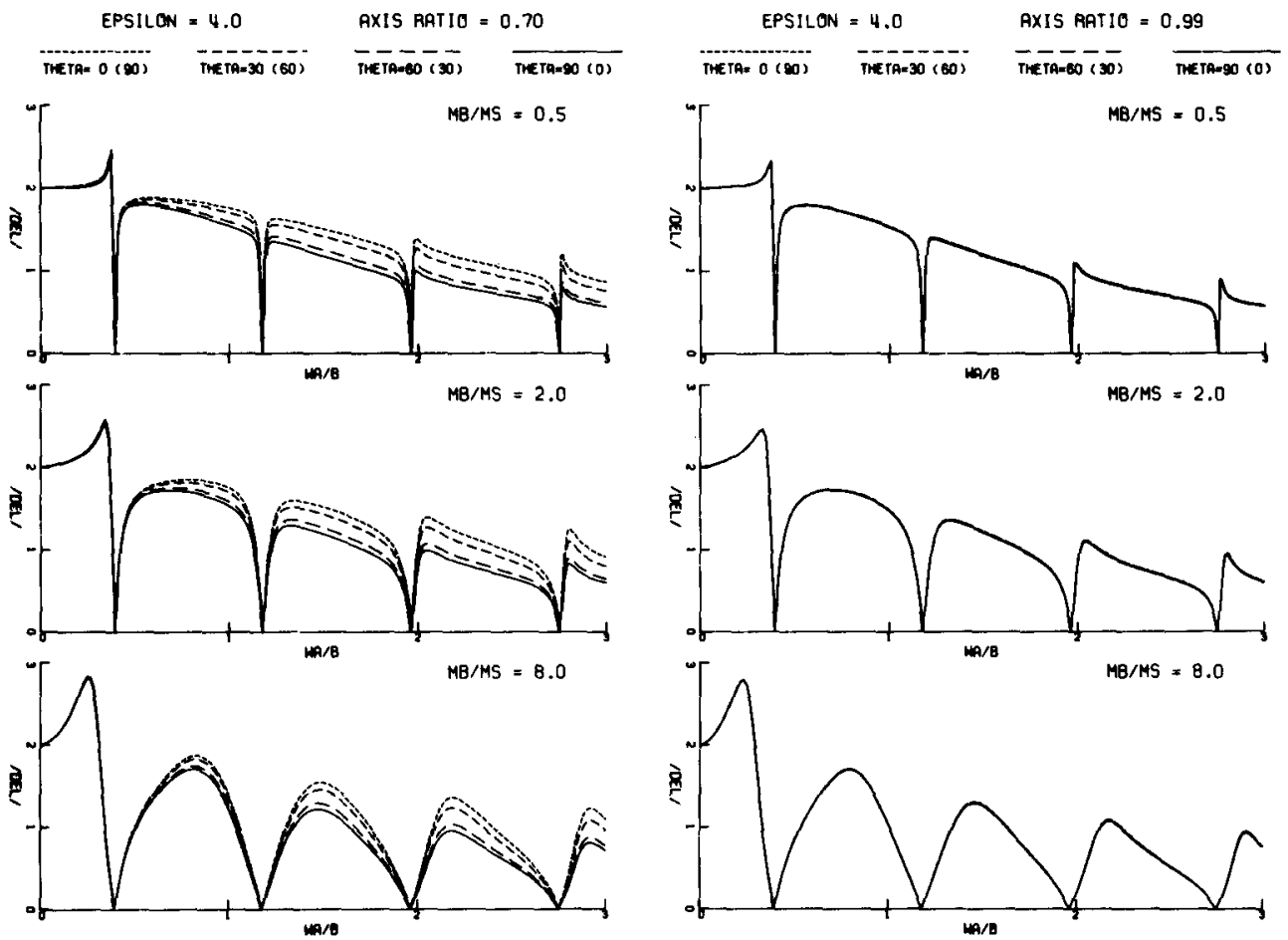

Fig. 5. Effect of interaction on the motion $|\Delta|$ of the rigid foundation. 


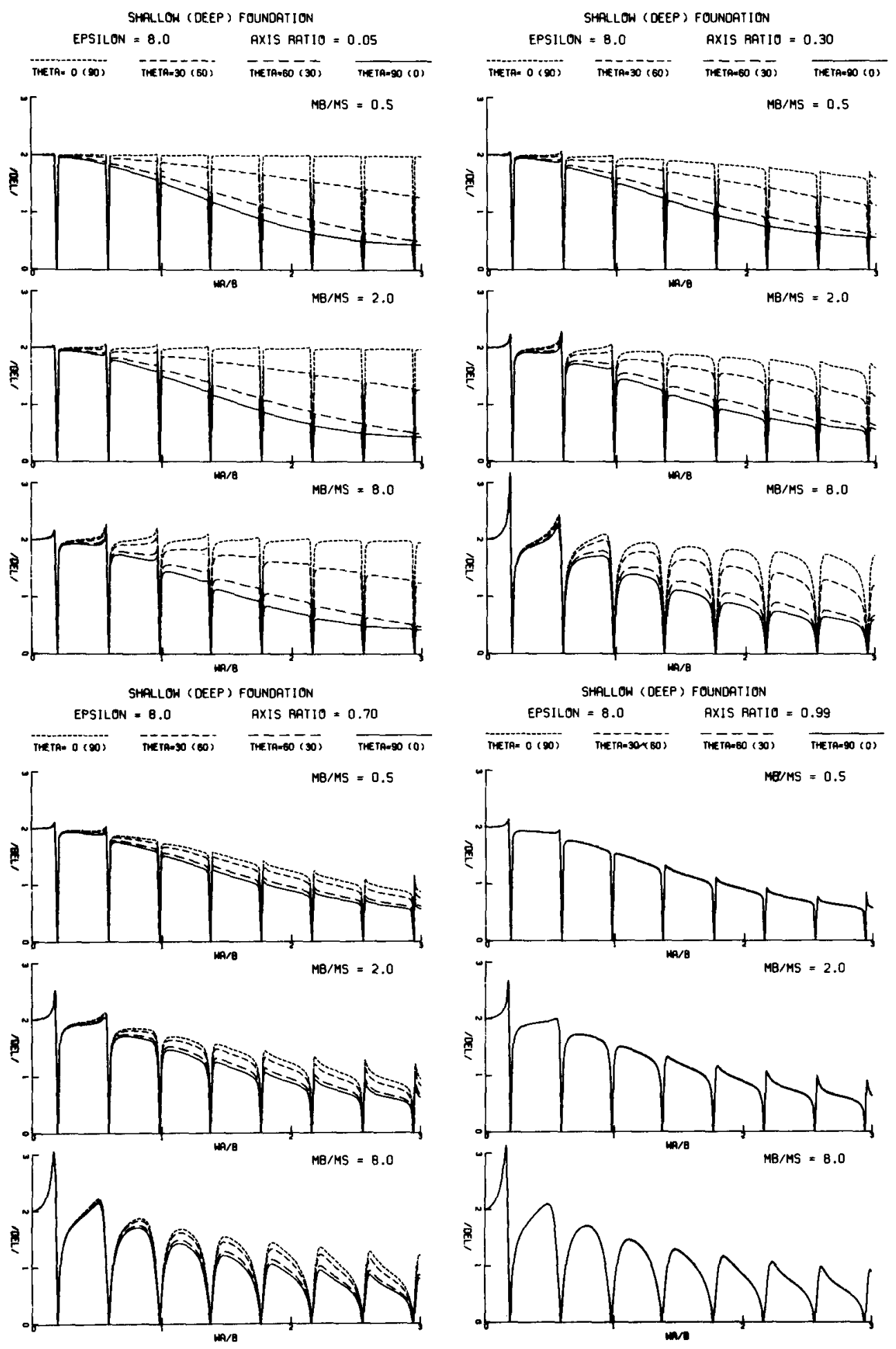

FIG. 6. Effect of interaction on the motion $|\Delta|$ of the rigid foundation. 
elastic spring and the equivalent dash-pot constants fixed, and then the increasing of $M_{b}$, which leads to the increase of $M=M_{S}+M_{0}+M_{b}$, results in the reduction of the equivalent natural frequency and the fraction of critical damping. This trend is clearly seen in Figure 2.

As $\varepsilon$ increases, the zeroes of $|\Delta|$ at $\omega A / \beta=\left(n+\frac{1}{2}\right) \pi / \varepsilon$ become smaller and more densely distributed, as may be seen in Figures 3 through 6 . The overall trends of the $|\Delta|$ versus $\omega A / \beta$ curves and the characteristics of the low-frequency peak which increases in amplitude with increasing $M_{b} / M_{S}$ remain the same as in Figure 2 for $\varepsilon=0$. The nature of THETA dependence on the shape of the foundation (axis ratio) and the rate at which $|\Delta|$ decreases with increasing $\omega A / \beta$ are also the same as those in Figure 2 . While $\varepsilon$ governs the position of zeroes in $|\Delta|$ diagrams, the width of the reduced $|\Delta|$ amplitudes centered around these zeroes increases with $M_{b} / M_{S}$. This behavior can be explained by noting the term $M_{b} / M_{S}\left(\tan k_{b} H / k_{b} H\right)$ in the denominator of equation (30). With larger $M_{b} / M_{S}$ this term affects the $|\Delta|$ in the wider interval of $\omega A / \beta$ centered around $\omega A / \beta=\left(n+\frac{1}{2}\right) \pi / \varepsilon$.

\section{Relative Response}

In the analysis and design of earthquake-resistant structures, it is necessary to know the maximum amplitudes of the displacement of the top of the structure relative to its foundation. From maxima of these relative displacements, it is possible to calculate the linear strains and therefore the maximum stresses experienced at any point in the structure. Using equation (23) for $x^{\prime}=H$, we calculate the relative response ${ }_{R} u_{z}$ at the top of the shear wall as

$$
\left|u_{z}\right|=\left|u_{z}\right|_{x^{\prime}=H}-\Delta|=| \Delta\left(\frac{1}{\cos k_{b} H}-1\right) \mid .
$$

When we neglect interaction, $\Delta$ would become 1 and $\left.\right|_{R} u_{z} \mid$ would reduce to

$$
\left|u_{z}\right|=\left|\frac{1}{\cos k_{b} H}-1\right| \text {. }
$$

From equation (27) it is seen that for the fixed base natural frequencies, $k_{b} H=\left(n+\frac{1}{2}\right)$ $\pi, n=1,2,3, \ldots$, the relative response given by (43) becomes infinite. However, if interaction is not neglected, since $\Delta$ is equal to zero when $k_{b} H=\left(n+\frac{1}{2}\right) \pi$, the relative response given by (42) remains finite. Thus, the interaction plays a role similar to the damping mechanisms, which are used to model the energy dissipation in the structural dynamics.

Figures 7,8, and 9 show the relative responses $\left.\right|_{R} u_{z} \mid$ given by equations (42) and (43) and for the same set of parameters used in describing $|\Delta|$ versus $\omega A / \beta$ in Figures 3 through 5 . The solid lines in these figures correspond to equation (43) and tend to infinity for $\omega A / \beta=\left(n+\frac{1}{2}\right) \pi / \varepsilon$. The dashed lines correspond to equation (42) and show the relative response for four typical incidence angles THETA $=0^{\circ}, 30^{\circ}, 60^{\circ}$, and $90^{\circ}$. It is seen that the relative response is strongly dependent on the incidence angle of $S H$ waves when the axis ratio of the elliptical rigid foundation is small. When the axis ratio tends to one, i.e., when the cross section of the foundation becomes circular, the THETA dependence disappears.

The ratio $M_{b} / M_{S}$ has a pronounced influence on the shape of the relative response curves (Figures 7, 8, and 9). As it increases, the overall amplitudes of the relative response decrease and change their shape appreciably. This change is so pronounced for the large values of $M_{b} / M_{S}$ that the peaks at $\omega A / \beta=\left(n+\frac{1}{2}\right) \pi / \varepsilon, n=1,2, \ldots$, corresponding to the fixed base frequencies, are completely lost. This could, therefore, represent one possible mechanism that might explain the differences between the calculated and measured natural frequencies for full scale structures. 

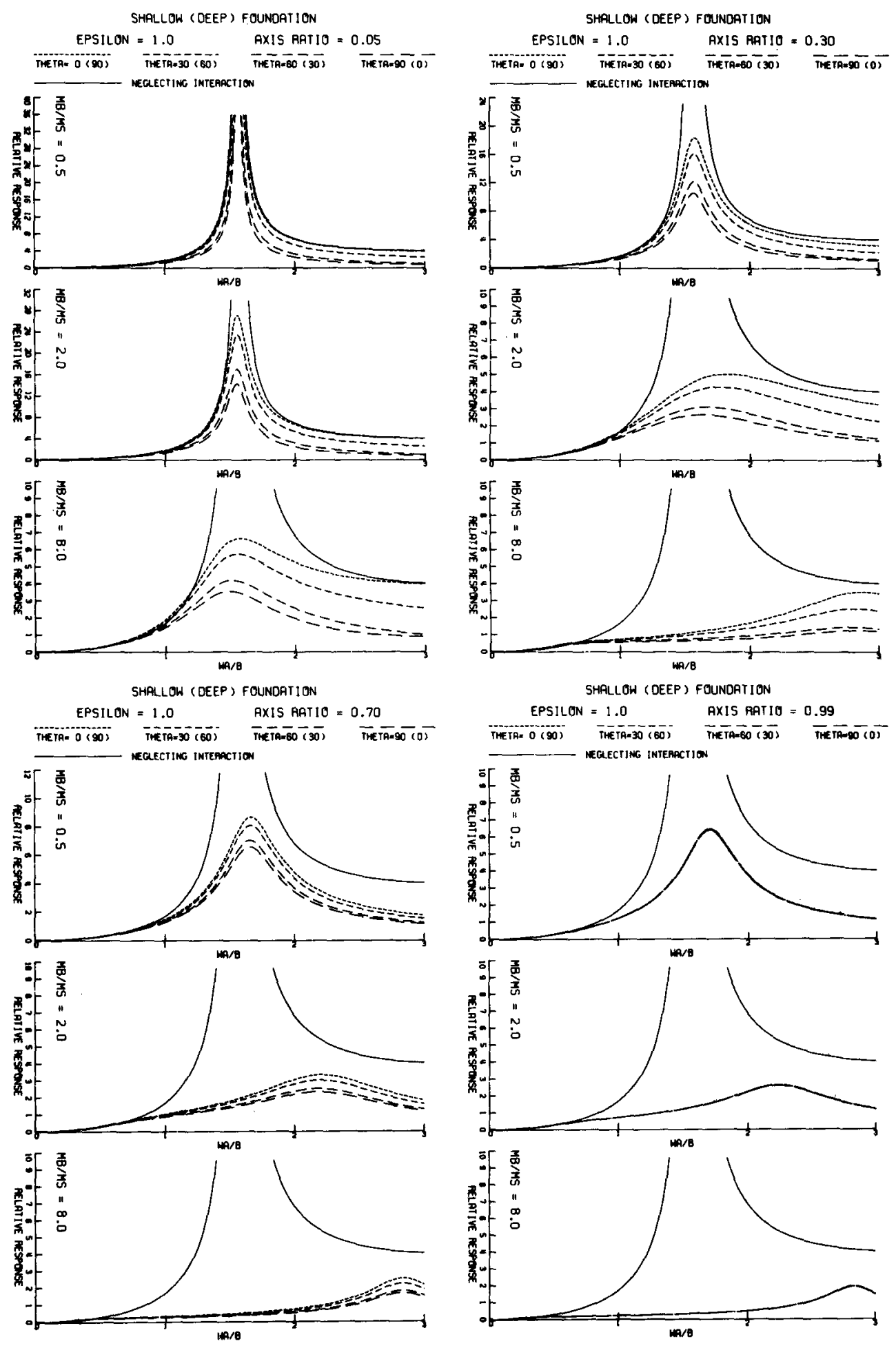

FIG. 7. Effect of interaction on relative response. 

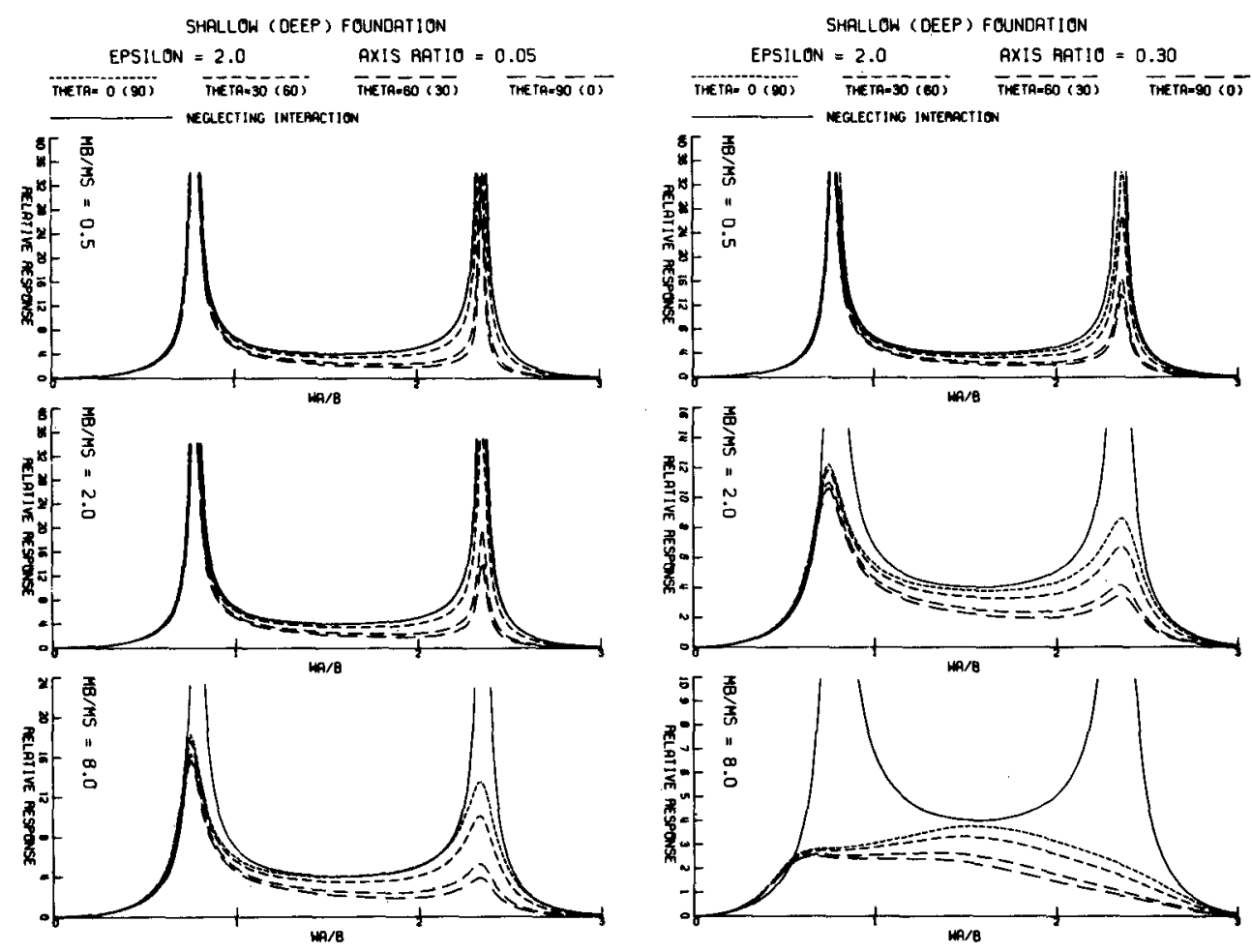

SHALLOW (DEEP) FOUNDAIION
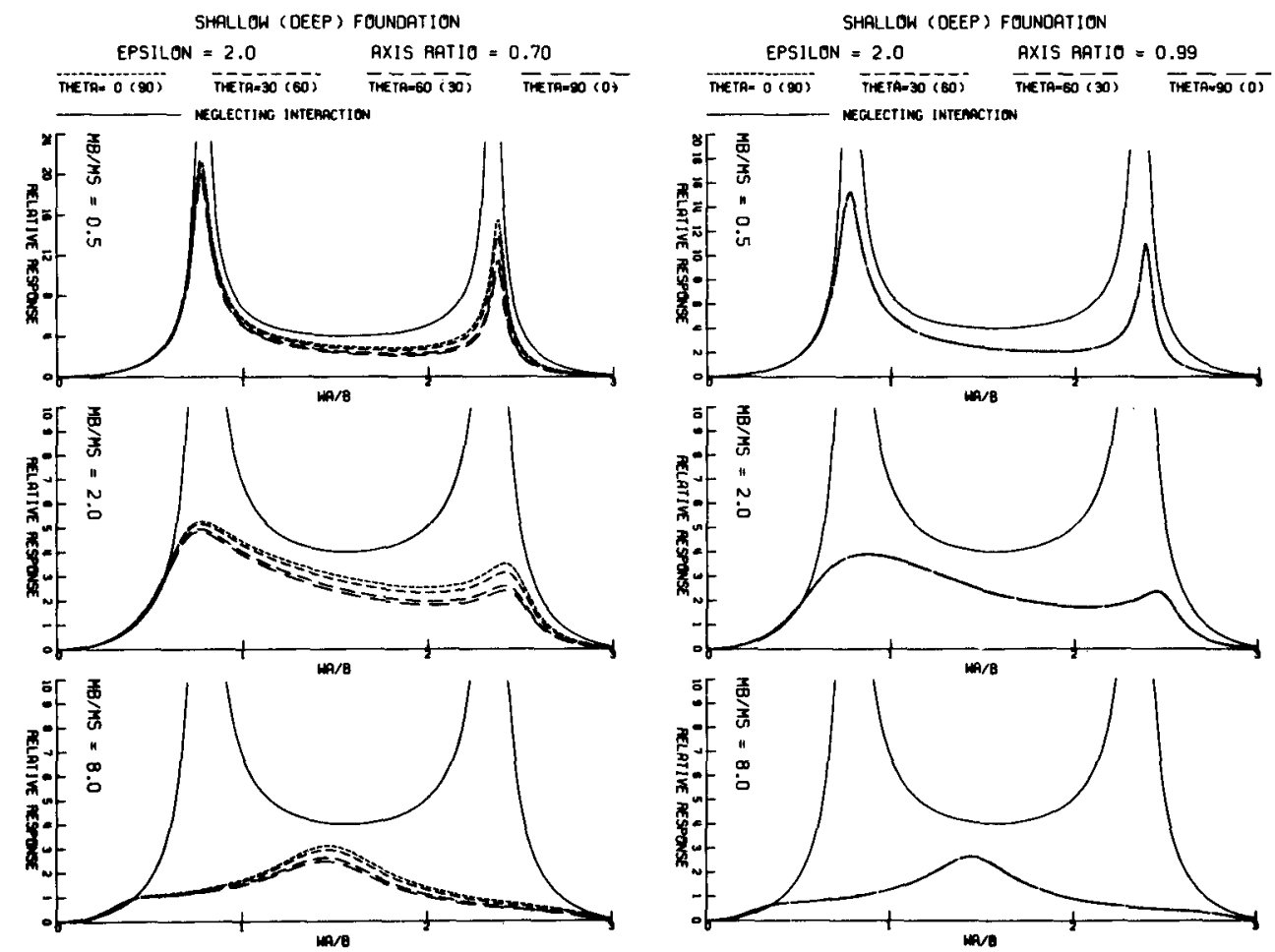

FIG. 8. Effect of interaction on relative response. 

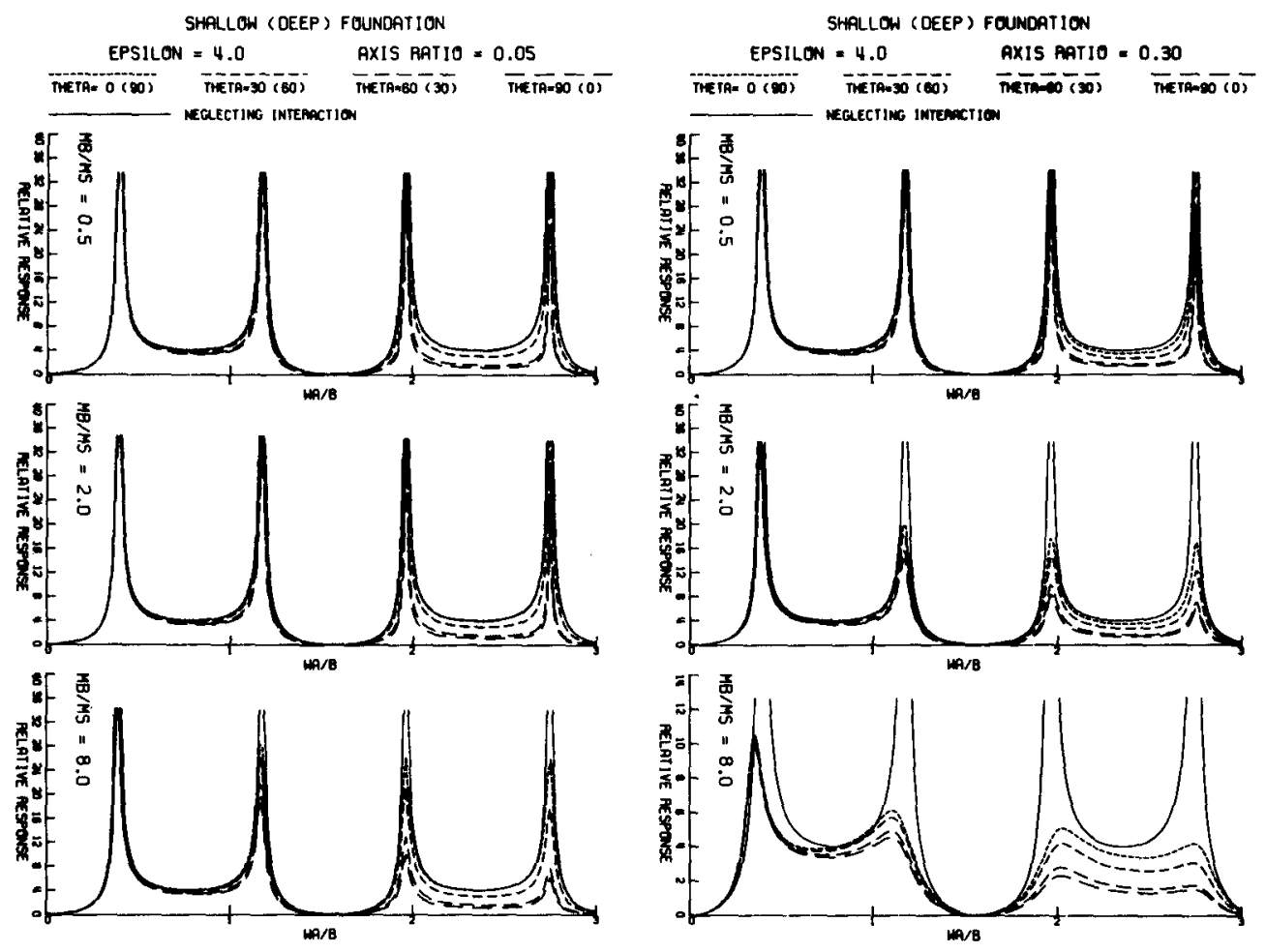

SHALLOW (DEEP) FOUNOATION

SHALLOH (DEEP) FOUNDRTION
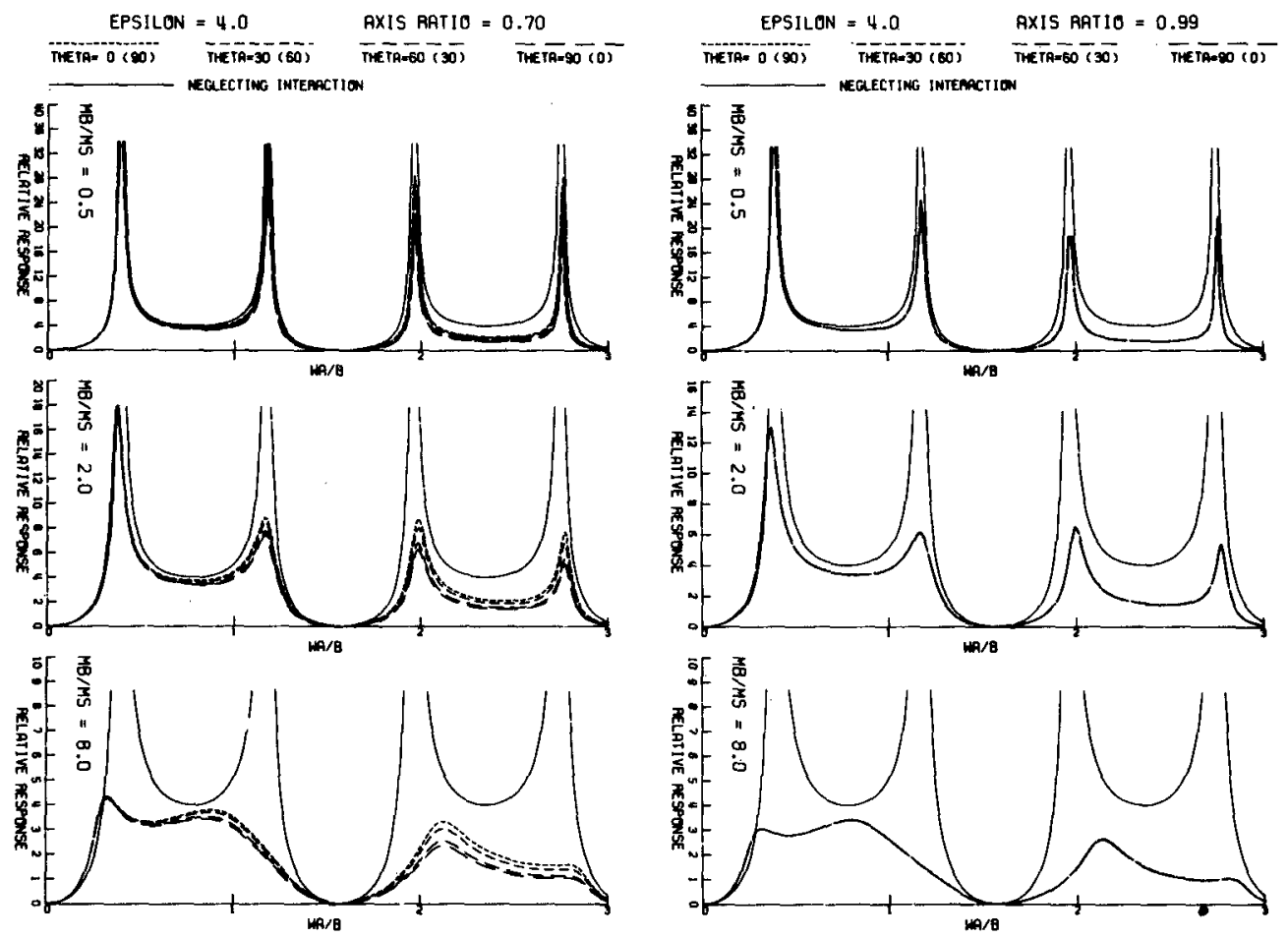

Fig. 9. Effect of interaction on relative response. 


\section{CONCLUSIONS}

In this paper, we have generalized the results for the two-dimensional dynamic soilstructure interaction problem, previously known only for the rigid semi-circular foundation (Luco, 1969; Trifunac, 1972), to the case of rigid foundation with the elliptical cross section. Although such a two-dimensional model might be used in the analysis of very long structures only, the exact nature of the solution gives a valuable insight into the physical nature of the problem. Of special interest in the analysis have been the effects of the foundation depth on the overall interaction amplitudes and the extent of coupling with the motion of the half-space for different angles of incident $S H$ waves. We found that the motion of the rigid foundation is more dependent on the angle of incidence for the foundation mass characterized by a small "minor-to-major axis ratio," and that when this ratio tends to one, this dependence on the angle of incidence disappears.

For the fixed-base natural frequencies of the undamped shear wall erected on top of the rigid foundation, the amplitude of the foundation motion is zero. Essentially for all other frequencies, this amplitude is less than the "free-field" amplitude of the half-space motion excited by the plane $S H$ waves and equal to 2 . The only exception to this occurs for a frequency band $0<\omega A / \beta<\pi / 2 \varepsilon$ when the amplitude of the foundation displacement may be larger than 2 . This amplification corresponds to the "natural frequency" of the rigid foundation embedded into the half-space and it increases with the increasing total mass of the foundation and the building. The frequency at which this amplitude occurs decreases with the increase of this total mass.

\section{ACKNOWLEDGMENTS}

We are indebted to P.C. Jennings and J. E. Luco for critical reading of the manuscript and several valuable comments.

This research has been supported by grants from the National Science Foundation and the Earthquake Research Affiliates Program at the California Institute of Technology.

\section{REFERENCES}

Abramovitz, M. A. and I. A. Stegun (1970). Handbook of Mathematical Functions, Dover, N.Y.

Luco, J. E. (1969). Dynamic interaction of a shear wall with the soil, J. Eng. Mech. Div., Am. Soc. Civil Engrs. 95, 333-346.

Luco, J. E. and R. A. Westman (1971). Dynamic response of circular footings, J. Eng. Mech. Div. Am. Soc. Civil Engrs. 97, EM5, 1381-1395.

Meixner, J. (1954). Mathieusche Funktionen und Sphäroidfunktionen, Springer-Verlag, Berlin.

Novak, M. (1973). The Effect of Embedment on Vibration of Footings and Structures, Proc. World Conf. on Earthquake Engr., 5th, Rome, Italy.

Oien, M. A. (1971). Steady motion of a rigid strip bonded to an elastic half space, J. Appl. Mech.38, Trans. ASME 93, 328-334.

Richart, F. E., Jr., J. R. Hall, Jr., and R. D. Woods (1970). Vibrations of Soils and Foundations, PrenticeHall, Englewood Cliffs, N.J.

Tajimi, H. (1969). Dynamic analysis of a structure embeded in an elastic stratum, Proc. World Conf. on Earthquake Engr., 4th Santiago, Chile.

Thau, S. A. (1971). Motion of a finite rigid strip in an elastic half space subjected to blast wave loading, Intern. J. Solids Struct. 7, 193-211.

Trifunac, M. D. (1972). Interaction of a shear wall with the soil for incident plane $S H$ waves. Bull, Seism. Soc. Am. 62, 63-83.

Earthquake Engineering Research Laboratory

California Institute of Technology

Pasadena, California 91109

Manuscript received March 29, 1974 